\title{
Review: lithium augmentation increases treatment response in refractory depression
}

Bauer M, Döpfmer S. Lithium augmentation in treatment-resistant depression: meta-analysis of placebo-controlled studies. J Clin Psychopharmacol 1999 Oct;19:427-34.

\section{QUESTION: In patients with refractory depression, is lithium augmentation clinically effective?}

\section{Data sources}

Studies were identified by searching Medline (1980 to June 1997) and the Cochrane Library and by scanning the references of published reviews and standard textbooks.

\section{Study selection}

Studies were selected if they were double blind, placebo controlled trials that involved patients who had not responded to conventional antidepressants; accepted, operationalised diagnostic criteria for depression were used; and outcome measures included acceptable criteria for assessing response.

\section{Data extraction}

2 reviewers independently assessed the quality of each study (Quality Assessment Scale by Detsky) and resolved differences by consensus. Data were extracted on study population, antidepressant treatment, lithium dose, treatment duration, response criteria, and treatment response.

\section{Main results}

9 randomised controlled trials (RCTs) involving 234 patients met the inclusion criteria. The mean age in 8 RCTs ranged from 37 to 54 years. Treatment duration ranged from 48 hours to 42 days. Quality scores ranged from $39 \%$ to $93 \% .3$ RCTs (quality scores $57 \%, 86 \%$, and $93 \%$ ) used a minimum dose of $800 \mathrm{mg} /$ day (or a dose sufficient to reach lithium serum concentrations $\geqslant 0.5$ $\mathrm{mEq} / \mathrm{l}$ ) for $\geqslant 2$ weeks. The combined results of these 3 RCTs showed that lithium augmentation led to a higher response rate (defined by using Hamilton Rating Scale for Depression or Short Clinical Rating Scale scores) than did placebo $\{p=0.002\} *$ (table). Results were similar when all 9 RCTs were included in the meta-analysis $\{\mathrm{p}<0.001\}^{*}$ (table). When RCTs were entered into a cumulative meta-analysis in the order of increasing dose, the effect was statistically significant at a dose of 600 to $800 \mathrm{mg} /$ day, and results did not change with higher doses. A cumulative meta-analysis of RCTs entered in the order of increasing treatment duration showed a statistically significant effect at 7 days.
For correspondence: Dr M Bauer, Psychiatry and Biobehavioral Sciences, University of California, Los Angeles, 300 UCLA Medical

Plaza, Suite 2330, Los Angeles, $C A$ 90095-6968, USA $\mathrm{Fax}+1310206$ 4310 . hium augmentation $v$ placebo for refractory depression (treatment

Treatment r
up to $42 d) \dagger$

\begin{tabular}{lllll} 
& \multicolumn{2}{c}{ Weighted event rates } & & \\
\cline { 2 - 3 } Studies & Lithium & Placebo & RBI (95\% Cl) & NNT (CI) \\
$\begin{array}{lllll}3 \text { studies with sufficient dose } \\
\text { and duration of lithium }\end{array}$ & $50 \%$ & $23 \%$ & $114 \%(23$ to 270$)$ & 4 (3 to 11$)$ \\
\hline All 9 studies & $46 \%$ & $17 \%$ & $125 \%(45$ to 251$)$ & 4 (3 to 6$)$ \\
\hline
\end{tabular}

†Abbreviations defined in glossary. Treatment duration was 4 to 6 weeks.

\#Event rates calculated from data in article and data supplied by author.

\section{Conclusion}

Lithium augmentation increases treatment response in patients with refractory depression.

*p values calculated from data in article and data supplied by author.

\section{COMMENTARY}

Despite the hundreds of thousands of patients who have been studied in clinical trials of antidepressants, little information exists about strategies for limited response to an antidepressant. Lithium augmentation is 1 of the first and best studied strategies for drug refractory depression, but some doubts have remained about its efficacy because of negative results in some studies. In part, these results were a product of the small sample sizes in individual studies. For example, the 9 RCTs described in the review by Bauer and Döpfmer involved a total of 234 patients, so the average number of patients per study was only 26 . Therefore, metaanalyses are important for detecting effects by pooling study outcomes.

This review confirms an earlier meta-analysis that showed that lithium augmentation is an effective strategy in non-responders to antidepressants. ${ }^{1}$ The cumulative metaanalysis procedure also provides some evidence about the dose and duration of lithium augmentation, showing that lithium should be given at therapeutic doses of 600 to $800 \mathrm{mg} /$ day for $\geqslant 1$ week.

These results are informative for clinicians when they evaluate treatment strategies for non-responders. The authors quite rightly recommend lithium augmentation as the strategy of choice for non-responders to conventional (tricyclic) antidepressants. However, because only 1 study focused on selective serotonin reuptake inhibitors, ${ }^{2}$ limited evidence exists to support lithium augmentation of newer antidepressants. Given the recent negative findings from RCTs of other augmentation strategies, ${ }^{3}{ }^{4}$ it is imperative that we do more studies to guide our decision making in drug refractory depression.

Raymond W Lam, MD University of British Columbia Vancouver, British Columbia, Canada

1 Austin MP, Souza FG, Goodwin GM. Br J Psychiatry 1991;159:510-14.

2 Baumann P, Nil R, Souche A, et al. J Clin Psychopharmacol 1996;16:307-14.

3 Landen M, Bjorling G, Agren H, et al. Clin Psychiatry 1998;59:664-8.

4 Perez V, Soler J, Puigdemont D, et al. Arch Gen Psychiatry 1999;56:375-9. 